\title{
Ocular Findings in Trisomy 13: Nasolacrimal Duct Stenosis Case Series*
}

\author{
Samiksha Fouzdar-Jain1 ${ }^{1}$, Yan Saoirse ${ }^{2}$, Brian Conahan ${ }^{3 \#, ~ D o n n y ~ S u h}{ }^{1}$ \\ ${ }^{1}$ University of Nebraska Medical Center and Children's Hospital of Omaha, Nebraska, USA \\ ${ }^{2}$ University of Nebraksa Medical Center, Omaha, Nebraska, USA \\ ${ }^{3}$ School of Medicine, Creighton University, Omaha, Nebraska, USA \\ Email: "bconahan31@gmail.com
}

How to cite this paper: Fouzdar-Jain, S., Saoirse, Y., Conahan, B. and Suh, D. (2019) Ocular Findings in Trisomy 13: Nasolacrimal Duct Stenosis Case Series. Open Journal of Ophthalmology, 9, 161-164.

https://doi.org/10.4236/ojoph.2019.94017

Received: September 9, 2019

Accepted: October 8, 2019

Published: October 11, 2019

Copyright (c) 2019 by author(s) and Scientific Research Publishing Inc. This work is licensed under the Creative Commons Attribution International License (CC BY 4.0).

http://creativecommons.org/licenses/by/4.0/

cc) (i) Open Access

\begin{abstract}
Trisomy 13, also known as Patau Syndrome, is a congenital malformation that leads to several ocular anomalies, of which cataracts are the most common, as well as iris and retinal colobomas, persistent hyperplastic primary vitreous, persistent tunica vasculosa lentis, and microphthalmos. While most do not survive beyond the neonatal period, those that do survive past one year of age have an $84 \%$ chance of making it to age 5 . Therefore, treatments for ocular problems should be used to improve the quality of life if the child can withstand surgery. The literature on this topic has described histopathologic findings in postmortem eyes. This case series supports those observations and presents a new discovery of nasolacrimal duct obstructions or stenosis. Deciding on surgical intervention in cases with Trisomy 13 can be challenging, and a variety of factors must be taken into account before surgery is considered. Treatment of these cases can be difficult, necessitating deliberate consideration and determination on a case-by-case basis; however, this case series gives additional evidence to help guide these decisions. Traditionally, trisomy 13 was regarded as being lethal, multiple organ malformations and severe intellectual disability.
\end{abstract}

\section{Keywords}

Trisomy 13, Nasolacrimal Duct, New Finding in Patau Syndrome

\section{Introduction}

Trisomy 13 is the third most commonly diagnosed autosomal trisomy in live-born patients, following trisomy 21 and trisomy 18, [1] with an incidence

*The parents of the children all signed a consent form allowing for the publication of this information. 
ranging from one in 5000 to one in 29,000 live births [2]. Traditionally, trisomy 13 was regarded as being lethal, multiple organ malformations and severe intellectual disability.

The prevalence of eye anomalies in Patau Syndrome is 30\% [3]. Common ocular manifestations include congenital cataract, iris coloboma, retina dysplasia, microphthalmos, and persistent hyperplastic primary vitreous (PHPV) [4]-[12]. Therapeutic approaches to these manifestations have a limited or guarded prognosis, in terms of visual outcomes, and active treatment options remain controversial. However, in recent years, with advancements in neonatal care, including inpatient care and surgery, as well as parents who are willing to pursue medical intervention, the survival rate was reported up to $50 \%$ at one year. Thus, stereotypes are being altered, and more patients are presenting to an ophthalmologist for further management to improve the quality of life.

Over the past few decades, minimal descriptions of clinical findings and interventions have been published, and detailed case studies emphasized pathologic abnormalities. This report describes a new clinical ocular finding of two patients with trisomy 13 that present with bilateral obstruction of the nasolacrimal duct.

\section{Case Report}

This case series describes two patients, both born premature, with Patient 1 born at 37 weeks and Patient 2 born at 39 weeks and two days. Patient 1 is an African-America male, born via cesarean section to a 19-year-old mother with pregnancy complications of intrauterine growth restriction (IUGR) and Group B streptococcus (GBS) colonization. Systemic abnormalities included a bicuspid aortic valve, patent ductus arteriosus, and ventricular septal defect. The patient had an eye exam at four months of age and showed a $9 \mathrm{~mm}$ cornea diameter persistent pupillary membrane, $3+$ posterior subcapsular cataract, and persistent hyperplastic primary vitreous (PHPV) in the right (OD) eye. The left (OS) eye had an enlarged cornea of $13 \mathrm{~mm}$ diameter and an intraocular pressure (IOP) of $54 \mathrm{mmHg}$, a goniotomy was performed to lower the pressure. An ultrasound of both eyes showed bilateral persistent hyaloid vessel. Cataract extraction with anterior vitrectomy of the right eye was performed, and an aphakic contact lens was used, which gradually improved vision. At 16 months of age, significant bilateral obstruction of the nasolacrimal duct was found, causing persistent epiphora. Two months later, probing and irrigation and punctoplasty of both eyes were performed, which resolved the problem significantly.

Patient 2 is an African-American female born via vaginal delivery to a 32-year-old mother whose pregnancy was complicated by gestational diabetes. Systemic findings include bilateral vocal cord paralysis, congenital laryngomalacia, cystic gallbladder, and a two-vessel umbilical cord. At 14 days of life, an eye exam was performed that revealed bilateral anterior polar cataracts and bilateral obstruction of the nasolacrimal duct, resulting in persistent eye drainage. Un- 
fortunately, the patient was not able to undergo any eye surgeries because follow-up was lost.

\section{Discussion}

Ocular malformation is one of the characteristic clinical features in patients with trisomy 13. Detailed case series of ocular findings were reported previously, which mostly focused on histopathologic findings in postmortem eyes, including iris colobomas, cataracts, retinal dysplasia, PHPV, and microphthalmos [10] [11] [12]. Lueder reported four patients with inferonasal iris coloboma and lens opacities in living patients [12]. This new finding of nasolacrimal duct obstruction as described in this case series has never been reported and can be found in early eye exams. It is essential to find common and relevant ocular abnormalities in patients with trisomy 13 , as there has been an increase in survival time, and surgeries can be done to improve the quality of life if the patient is healthy enough to survive the surgery. Secondary eye infections due to nasolacrimal duct obstruction is a nuisance, but an early nasolacrimal duct probing and irrigation can decrease complication. The goal for patients with trisomy 13 is to improve their quality of life, so surgery should not be performed if the patient will not survive surgery. However, it is reasonable to consider ocular surgeries if the problem is treatable and will improve. Treatment in trisomy 13 is very challenging, requiring it to be handled on a case-by-case basis. Therefore, a deliberate conversation should be had with parents regarding surgeries that could be done to improve the quality of life, including those that could improve vision.

\section{Summary}

In summary, this new finding of nasolacrimal duct obstruction will help guide further ocular findings in patients with trisomy 13, leading to an improvement in the quality of life of patients.

\section{Conflicts of Interest}

The authors declare no conflicts of interest regarding the publication of this paper.

\section{References}

[1] Parker, S.E., Mai, C.T., Canfield, M.A., Rickard, R., Wang, Y., Meyer, R.E., Anderson, P., Mason, C.A., Collins, J.S., Kirby, R.S. and Correa, A. (2010) Updated National Birth Prevalence Estimates for Selected Birth Defects in the United States, 2004-2006. Birth Defects Research Part A: Clinical and Molecular Teratology, 88, 1008-1016. https://doi.org/10.1002/bdra.20735

[2] Tsukada, K., Imataka, G., Suzumura, H. and Arisaka, O. (2012) Better Prognosis in Newborns with Trisomy 13 Who Received Intensive Treatments: A Retrospective Study of 16 Patients. Cell Biochemistry and Biophysics, 63, 191-198. https://doi.org/10.1007/s12013-012-9355-0

[3] Smith, D.W., Patau, K., Therman, E., Inhorn, S.L. and DeMars, R.I. (1963) The D1 
Trisomy Syndrome. The Journal of Pediatrics, 62, 326-341. https://doi.org/10.1016/S0022-3476(63)80129-8

[4] Patau, K., Smith, D.W., Therman, E., et al. (1960) Multiple Congenital Anomalies Caused by an Extra Autosome. The Lancet, 1, 790-793.

https://doi.org/10.1016/S0140-6736(60)90676-0

[5] Cogan, D.G. and Kuwabara, T. (1964) Ocular Pathology of the 13-15 Trisomy Syndrome. Archives of Ophthalmology, 72, 246-253. https://doi.org/10.1001/archopht.1964.00970020246021

[6] Keith, C.G. (1966) The Ocular Manifestations of Trisomy 13-15. Transactions of the Ophthalmological Societies of the United Kingdom, 86, 435-454.

[7] Ginsberg, J. and Perrin, E.V.D. (1965) Ocular Manifestations of 13-15 Trisomy. Archives of Ophthalmology, 74, 487-495. https://doi.org/10.1001/archopht.1965.00970040489010

[8] Hoepner, J. and Yanoff, M. (1972) Ocular Anomalies in Trisomy 13-15: An Analysis of 13 Eyes with Two New Findings. American Journal of Ophthalmology, 74, 729-737. https://doi.org/10.1016/0002-9394(72)90836-7

[9] Allen, J.C., de Venecia, G. and Opitz, J.M. (1977) Eye Findings in the 13 Trisomy Syndrome. European Journal of Pediatrics, 124, 179-183. https://doi.org/10.1007/BF00452109

[10] Koole, F.D., Velzeboer, C.M.J. and Van der Harten, J.J. (1990) Ocular Abnormalities in Patau Syndrome. Ophthalmic Paediatrics and Genetics, 11, 15-21. https://doi.org/10.3109/13816819009012944

[11] Magni, R., Pierro, L. and Brancato, R. (1991) Microphthalmos with Colobomatous Orbital Cyst in Trisomy 13. Ophthalmic Paediatrics and Genetics, 12, 39-42. https://doi.org/10.3109/13816819109023083

[12] Meyer, R.E., Liu, G., Gilboa, S.M., Ethen, M.K., Aylsworth, A.S., Powell, C.M., Flood, T.J., Mai, C.T., Wang, Y. and Canfield, M.A. (2016) Survival of Children with Trisomy 13 and Trisomy 18: A Multi-State Population-Based Study. American Journal of Medical Genetics Part A, 170, 825-837.

https://doi.org/10.1002/ajmg.a.37495 